Recherches en didactique des langues et des cultures

Les cahiers de l'Acedle

\title{
Linguistique et didactique : où en est-on ? Quelle place pour une approche conceptualisante de la construction de la langue dans la perspective actionnelle?
}

Danielle Chini

\author{
(2) OpenEdition \\ Journals \\ Édition électronique \\ URL : http://journals.openedition.org/rdlc/1958 \\ DOI : $10.4000 /$ rdlc. 1958 \\ ISSN : 1958-5772 \\ Éditeur \\ ACEDLE
}

Référence électronique

Danielle Chini, «Linguistique et didactique : où en est-on ? Quelle place pour une approche conceptualisante de la construction de la lanque dans la perspective actionnelle? ", Recherches en didactique des langues et des cultures [En ligne], 6-2 | 2009, mis en ligne le 01 octobre 2009, consulté le 01 mai 2019. URL : http://journals.openedition.org/rdlc/1958 ; DOI : 10.4000/rdlc.1958

Ce document a été généré automatiquement le 1 mai 2019.

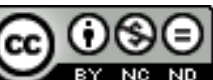

Recherches en didactique des langues et des cultures is licensed under a Creative Commons AttributionNonCommercial-NoDerivatives 4.0 International License 


\title{
Linguistique et didactique : où en est-on? Quelle place pour une approche conceptualisante de la construction de la langue dans la perspective actionnelle?
}

\author{
Danielle Chini
}

\section{Introduction}

1 Si la question de la nature des relations entre didactique et linguistique n'est pas nouvelle, entre application, transposition, implication, corrélation..., c'est bien une question complexe à laquelle il serait présomptueux de prétendre apporter une réponse définitive, ne serait-ce qu'à cause du renouvellement des données du problème qu'entraîne chaque réorientation méthodologique majeure, l'adossement au Cadre européen commun de référence (désormais CECR; Conseil de l'Europe, 2001) n'ayant, de ce point de vue, pas dérogé à la règle. Tout dépend de la tradition disciplinaire d'où on est issu (autrement dit de la langue qu'on enseigne), du public que l'on considère, institutionnel ou pas, scolaire ou pas, enfant ou adulte, etc., ainsi que du regard "linguistique" que l'on adopte - celui de la linguistique théorique, celui de la psycholinguistique, ou de la sociolinguistique, ou encore celui d'une des nombreuses autres "sciences" dites "du langage"... J'affirmerai donc en préambule que les remarques, forcément subjectives, qui suivent sont celles d'une didacticienne angliciste, tout à la fois engagée dans la formation des enseignants du secondaire et linguiste énonciativiste, qu'elles émanent d'une évaluation des questions que soulève dans ce champ la mise en place de la perspective actionnelle, et qu'elles prolongent de nombreux travaux antérieurs consacrés à l'étude de la problématique linguistique de l'enseignement- 
apprentissage de l'anglais, appréhendée au travers d'une lecture critique des orientations et textes officiels (cf., entre autres, Chini, 1997a, 1997b, 2004, 2008a, 2009a).

\section{CECR et DLE : quel positionnement linguistique?}

2 Du point de vue des relations entre linguistique et didactique, l'adossement au CECR me semble avoir entraîné une évolution significative des attitudes dominantes au sein de la Didactique des langues étrangères (maintenant DLE), qui se caractérise par une centration marquée sur des problématiques sociolinguistiques ${ }^{1}$, entre autres la question de la norme et de la variation, souvent abordée par la stigmatisation de l'une et l'affirmation de la primauté de l'autre, en particulier dans une perspective de recherche de valorisation de la dynamique actionnelle et plurilingue. Ainsi lors la journée $\mathrm{NeQ}$ sur les plurilinguismes qui s'est tenue à Tours en janvier 2009 (journée organisée à l'université François Rabelais par l'Acedle et le laboratoire Dynadiv, textes à paraître), Didier de Robillard, sociolinguiste créoliste, a, à partir d'une critique de l'expression "la langue en soi", développé une argumentation qui stigmatisait le rôle néfaste de la " linguistique dominante", qui aurait créé de toutes pièces le concept de langue, conçu comme un système stable et clos sur lui-même, ignorant ainsi toute dimension historique et dynamique et l'instaurant comme objet normé, alors même que pour lui, dans ce domaine, "l'instabilité est constitutive et fonctionnelle" et tout n'est que procédures, comme le reconnait, en revanche, la sociolinguistique. Dans cette perspective, la variation est donc première et les langues ne peuvent être que des "émulsions de plein de choses", des " précipités" qui, comme l'être humain sont "imprédictibles". En conséquence, pour lui, la question se poserait même de savoir si elles sont "enseignables et apprenables" (citations transcrites de l'enregistrement vidéo de l'intervention, voir de Robillard, 2009). Au-delà de son caractère sans nuances ${ }^{2}$, cette intervention, ne serait-ce que par le fait qu'elle ait eu sa place dans une journée d'études en DLE, me semble en effet significative de cette évolution. D'ailleurs une recherche rapide et non exhaustive révèle que ce thème, variation vs norme, fait florès puisque de nombreux colloques ou publications viennent ou vont lui être consacrés ${ }^{3}$, tant en didactique qu'en linguistique.

3 Il ne s'agit ici d'ignorer ni le caractère traditionnellement normatif de la grammaire scolaire, prioritairement ancrée dans la langue écrite, ni la réalité de la variation, qui, elle, est inhérente à l'usage quotidien, surtout oral, de la langue, et encore moins de nier la nécessité d'une prise en compte de la sociolinguistique pour la compréhension des pratiques, qu'il s'agisse de celles de la classe ou des pratiques sociales de référence. Cependant cette orientation, étroitement articulée au principe central de pilotage par la tâche, qui vise une efficacité à court terme en contexte social, me semble avoir un effet secondaire fort problématique, du moins en milieu scolaire, à savoir la mise à l'arrièreplan des questionnements sur la structuration cognitive et psycholinguistique des compétences linguistiques et langagières, appréhendée dans une logique constructiviste, questionnements qui étaient pourtant centraux pour la DLE lors de la décennie précédente, par le biais en particulier de l'importance accordée à la réflexion sur la langue (cf. l'instauration par les textes officiels de 1985 de la pratique raisonnée de la langue comme objectif fondamental), ou encore à l'ampleur prise par l'objectif méthodologique (cf. Chini, 2008b) lors de la rénovation des lycées et la création de l'enseignement modulaire, à partir de 1992 (cette orientation est inscrite dans l'expression consacrée pour désigner cette période de l'approche communicative, à savoir 
"l'approche communicative et cognitive"). Si ces objectifs n'ont pas été officiellement supprimés par les textes programmatiques parus depuis 2002, ils sont aujourd'hui très nettement passés au second plan, ne serait-ce que par manque de temps à y consacrer dans les classes (outre la réduction des horaires à deux heures dans la plupart des classes de lycée, il faut bien admettre que la perspective actionnelle est chronophage, ne seraitce que du fait de la place importante qu'y prend l'évaluation individuelle des compétences orales). Le corollaire en est, de mon point de vue, un recul significatif de l'influence de la linguistique, au sens étroit du terme. La quasi-disparition des références aux concepts énonciatifs qui avaient été introduits dans les années quatre-vingt-dix par le programme grammatical pour l'anglais en collège le prouve.

En effet, avant d'aller plus loin, je souhaiterais préciser le sens que je donne ici au terme "linguistique". À l'instar de la définition proposée par Henri Portine (2009) ici même (l'ensemble des références à cet auteur renvoient à sa contribution dans ce même numéro des Cahiers de l'Acedle), nombre de recherches actuelles en DLE s'inscrivent dans une conception large de la linguistique, comprise au sens anglo-saxon du terme, et correspondant dans la tradition française au champ ouvert des sciences du langage, parmi lesquelles Henri Portine classe d'ailleurs la didactique des langues, qui devient de facto une des composantes de la "linguistique"4. Si toutes les composantes de ce champ ont, n'en doutons pas, quelque chose à apporter à la didactique des langues, dans la mesure où elles traitent toutes des facettes diverses d'une même entité, je continuerai, pour ma part, sans nier l'utilité des autres approches et la nécessité absolue de croisements et d'analyses pluridisciplinaires, à situer ma réflexion par rapport à un champ plus étroit, celui de la linguistique dite "théorique", définie non pas comme une étude formelle de "l'outil-langue", mais comme une réflexion sur les relations formes / sens telles qu'elles se manifestent au sein du discours, à des fins de mise au jour de la cohérence interne des systèmes linguistiques, pour chaque langue évidemment, mais surtout au-delà, de façon transversale, et idéalement universalisante, l'objet véritable étant la compréhension de la dynamique langagière telle qu'elle se met en œuvre sur le plan des opérations cognitives et psycholinguistiques. S'il est indispensable, en effet, d'appréhender la question didactique d'un point de vue pragmatique et sociolinguistique, indissociable d'un entraînement efficace aux pratiques langagières, il me semble que ces questionnements n'auront de sens que s'ils s'articulent sur une prise en compte de la dynamique psycholinguistique (l'inverse étant d'ailleurs tout aussi vrai) qui fonde l'implication du sujet tout à la fois dans l'activité langagière et dans l'activité d'apprentissage. Or cela ne me semble pas être réellement le cas dans les conceptions méthodologiques actuelles découlant de la perspective actionnelle.

\section{Quelles conséquences méthodologiques?}

Certes, comme l'explique Henri Portine, "une méthodologie ne peut traiter de tous les aspects des apprentissages. Il faut donc procéder à des focalisations thématiques". Il ajoute :

un apprenant doit à la fois structurer correctement ses énoncés (correction/ accurracy) et se préparer à agir en temps réel (habilité langagière ou fluency), mais il ne peut pas faire les deux en même temps, thématiquement, il faut donc subordonner l'un à l'autre.

6 Autrement dit, on doit choisir entre les deux pôles qui, Henri Portine le rappelle, délimitent la linguistique, "le pôle formel" et "le pôle anthropologique", la didactique pouvant 
donc faire le choix d'entrer prioritairement dans la problématique de l'apprentissage soit par la langue, soit par les pratiques.

7 Le choix anthropologique de la perspective actionnelle est clair : l'entrée se fait par les pratiques, le sujet étant vu avant tout comme un acteur social et l'apprentissage n'étant qu'un des usages possibles de la langue. C'est en fait par le biais de la confrontation avec les aléas du processus de gestion collective et collaborative de la tâche que les connaissances et compétences sont censées se modifier, s'adapter, dans une sorte de boucle rétroactive, mais sans avoir à se centrer explicitement et spécifiquement sur l'apprentissage lui-même, qui est censé être un effet induit du pilotage par la tâche. Ceci apparait clairement dans la définition de la perspective actionnelle que propose le CECR (2001 : 15), sous le titre "Caractéristiques de toute forme d'usage et d'apprentissage d'une langue ".

L'usage d'une langue, y compris son apprentissage, comprend les actions accomplies par des gens qui, comme individus et comme acteurs sociaux, développent un ensemble de compétences générales et, notamment une compétence à communiquer langagièrement. Ils mettent en oeuvre les compétences dont ils disposent dans des contextes et des conditions variés et en se pliant à différentes contraintes afin de réaliser des activités langagières permettant de traiter (en réception et en production) des textes portant sur des thèmes à l'intérieur de domaines particuliers, en mobilisant les stratégies qui paraissent le mieux convenir à l'accomplissement des tâches à effectuer. Le contrôle de ces activités par les interlocuteurs conduit au renforcement ou à la modification des compétences.

On relèvera particulièrement la dernière phrase (les termes en gras sont dans le texte initial). Ainsi même si, dans le chapitre concernant "L'utilisation de la langue et l'apprenant / utilisateur", le CECR aborde bien la question des "stratégies", terme qui apparaît même dans le titre de la sous-partie centrale où l'on trouve les tableaux de descripteurs, il ne s'agit pas des stratégies d'apprentissage mais bien des stratégies de gestion de la tâche actionnelle, ce qui est très différent. Cette notion est définie ainsi (2001: 48).

Les stratégies sont le moyen utilisé par l'usager d'une langue pour mobiliser et équilibrer ses ressources et pour mettre en oeuvre des aptitudes et des opérations afin de répondre aux exigences de la communication en situation et d'exécuter la tâche avec succès et de la façon la plus complète et la plus économique possible - en fonction de son but précis.

On peut voir l'utilisation de stratégies communicatives comme l'application des principes métacognitifs : Pré-planification, Exécution, Contrôle et Remédiation des différentes formes de l'activité communicative: Réception, Interaction, Production et Médiation.

On a pu utiliser le mot "stratégies" avec des sens différents. On l'entend ici comme l'adoption d'une ligne de conduite particulière qui permet l'efficacité maximum. Les aptitudes qui constituent une partie inévitable des opérations de compréhension et d'articulation du mot écrit et parlé (par exemple, découper la chaîne parlée en mots significatifs) sont traitées comme des aptitudes de niveau inférieur, relatives au processus communicatif approprié.

Le progrès dans l'apprentissage d'une langue apparaît le mieux dans la capacité de l'apprenant à s'engager dans une activité langagière observable et à mettre en oeuvre des stratégies de communication. En conséquence, elles constituent une base pratique pour l'étalonnage de la capacité langagière. On trouvera dans ce chapitre une proposition d'étalonnage pour différents aspects d'activités et de stratégies qui seront commentées. 
Même s'il est beaucoup plus abouti et sophistiqué que les différentes versions du Threshold Level qui l'ont précédé, le CECR se situe dans la continuité du principe de l'analyse de besoins, c'est-à-dire qu'il part de l'objectif social à atteindre, des résultats visés, autrement dit, la réalisation rapide et efficace de la tâche spécifique, le cognitif n'étant, je cite, qu'une "partie inévitable des opérations" qui sera traitée comme "des aptitudes de niveau inférieur", et le linguistique n'intervenant qu'en fin de chaîne, lors de l'évaluation des moyens nécessaires à l'action, ne pouvant donc jouer qu'un rôle instrumental. Qui plus est, alors que le Threshold Level, d'inspiration pragmatique, restait dans le cadre d'une analyse des besoins langagiers et des actes de parole, l'approche actionnelle, en se situant prioritairement non plus par rapport à une théorie du langage mais par rapport à une théorie générale de l'action, qui fait des actes de paroles des actes comme les autres contribuant à l'accomplissement de tâches en contexte social, l'approche actionnelle, donc, élargit le champ. Et si, pour reprendre les termes de Henri Portine, la focalisation thématique de l'approche communicative classique portait bien sur le "rapport apprenant-apprenant" en visant une "modélisation du monde", la perspective actionnelle me semble plus relever d'une focalisation sur "le rapport apprenant-monde" qui vise "à réinscrire la classe dans le monde", ce qui tend à neutraliser la spécificité de l'apprentissage langagier, tout particulièrement en milieu scolaire contraint.

\section{Prise en compte de la spécificité du cadre scolaire}

10 Le milieu scolaire, en effet, se définit par le caractère essentiellement fictionnel des pratiques en langue étrangère, une temporalité extensive et fragmentée, ainsi que par un cadre situationnel contraint et pauvre (pauvre, bien sûr, du seul point de vue de la pratique langagière en langue étrangère à visée communicative ou actionnelle), ce qui induit une implication non spontanée des sujets dans l'échange. Qu'on le veuille ou non, ces caractéristiques le situent forcément en décalage avec les situations et les pratiques sociales de référence, aussi important que soit leur rôle de modèle. C'est bien ce que Rémy Porquier et Bernard Py (2004: 59) illustrent lorsqu'ils distinguent deux niveaux d'interaction dans l'appropriation d'une langue, un "contexte macro" où interviennent les déterminants sociaux, et le "contexte micro" de la salle de classe. Or si l'approche communicative classique prenait en compte cette réalité et acceptait d'emblée le principe de simulation, la perspective actionnelle semble vouloir sauter le pas de l'utopie naturaliste, en prétendant faire de l'élève "un acteur social, usager de la langue, ayant à accomplir des tâches (qui ne sont pas seulement langagières)" (CECR : 15) et qui ouvrent sur un résultat concret à court terme.

11 En fait, ce qui pose problème, c'est la volonté déclarée du CECR de constituer un cadre commun pour l'ensemble des situations d'apprentissage, même s'il se veut non dogmatique, et donc adaptable à toutes les situations et toutes les traditions éducatives. De là d'ailleurs découle son refus de se situer, en termes d'apprentissage, par rapport à quelque théorie linguistique ou cognitive que ce soit, laissant les décideurs nationaux ou locaux libres de la logique préconisée, et laissant ainsi grande ouverte la porte de l'éclectisme, même s'il n'est pas revendiqué. Mais, comme depuis les premiers travaux du Conseil de l'Europe, la réflexion part d'une analyse des comportements langagiers visés, c'est-à-dire des caractéristiques des pratiques langagières en milieu naturel, voire des processus acquisitionnels en milieu endolingue motivé, considérations par bien des aspects peu adaptées aux contraintes du cadre scolaire traditionnel. Cette tendance à la 
neutralisation de la spécificité scolaire, dont se préoccupait pourtant traditionnellement la didactique, est clairement marquée par l'importance actuellement accordée aux situations d'apprentissage non institutionnelles, comme les actions de formation tout au long de la vie (cf. le thème du prochain colloque de l'Acedle, "Les langues tout au long de la vie") qui posent des problèmes différents dans la mesure où, pour la plupart, elles s'adressent à un public d'adultes, soit volontaires, soit motivés par un objectif ciblé à court terme, de nature professionnelle ou sociale, ou encore comme la grande variété des situations d'apprentissage du Français Langue Étrangère, qui peut se dérouler en contexte endolingue aussi bien qu'exolingue, et avec un public divers, souvent migrant, pour lequel le français prend alors un statut non de langue étrangère, mais de langue seconde, le travail dans la classe étant prolongé par la pratique sociale et interactive quotidienne. Dans de telles conditions, la nécessité d'efficacité immédiate, le contexte acquisitionnel ainsi que parfois le cadre intensif de la formation, viennent considérablement modifier les données, et la perspective actionnelle, qui valorise l'agir social collaboratif, peut se révéler être une réponse pertinente.

En milieu scolaire, comme nous l'avons vu, les conditions sont tout autres: le véritable objectif social y est l'appropriation et donc la construction de la langue étrangère, certes prioritairement comme moyen de l'action sociale, mais cette portée concrète n'est réellement appréhendable qu'à moyen ou long terme. Et, pas toujours certes, mais souvent, les tâches dites actionnelles que l'on peut y concevoir ne sont, par manque d'ancrage authentique hors du cadre scolaire, ainsi que, souvent, par compréhension insuffisante de la philosophie actionnelle par les enseignants (ou les concepteurs de manuels), que des simulations communicatives déguisées, mais rendues envahissantes par la doxa de la tâche, et surtout par le refus de prendre réellement en compte le caractère de théâtralisation du contexte scolaire ${ }^{5}$.

Pourtant c'est bien la mise à distance réflexive que permet cette nature "théâtrale" des tâches en milieu scolaire qui constitue la spécificité de la classe comme lieu social. C'est, en d'autres termes, ce que les didacticiens du français langue maternelle nomment, à la suite de Jean-Paul Bronckart (1996), la secondarisation des discours. Ce "second niveau", explique cet auteur (2001: 143), qui se surajoute au premier où se développe la praxis, est celui des "évaluations langagières [qui] portent inévitablement sur les modalités de participation des individus aux activités collectives" et servent au contrôle du processus didactique. JeanPaul Bernié (2003 : 29) voit cette secondarisation comme

un outil majeur pour penser le langage dans la classe (...) qui permet de lui attribuer un rôle important dans l'autonomisation des concepts par rapport à leur formulation initiale et dans la construction d'une posture disciplinaire adéquate.

C'est là que se noue la véritable relation sociale de la classe, la relation de médiation pédagogique, qu'elle s'établisse entre l'enseignant et les élèves dans leur rôle d'apprenants ou, de façon collaborative, entre les apprenants eux-mêmes. C'est là que les pratiques interactives, qu'elles soient communicatives ou actionnelles, peuvent devenir ce que nos collègues suisses (Cf. par exemple de Pietro, Matthey \& Py, 1989 ou de Pietro \& Schneuwly, 1997) ont qualifié de "séquences potentiellement acquisitionnelles", à condition qu'elles ne portent pas exclusivement sur la gestion de la tâche spécifique, mais sur les processus récurrents en jeu dans l'utilisation de la langue, qu'il s'agisse des processus cognitifs et psycholinguistiques qui sous-tendent les compétences langagières ou, bien sûr, de l'analyse des relations formes / sens, et des opérations non aléatoires qu'elles 
révèlent petit à petit, en relation avec des classes de situations, qu'on les qualifie de pragmatiques ou d'actionnelles.

Il ne s'agit bien entendu pas de revenir à un enseignement de la langue pour la langue et de nier l'importance qu'il y a à rechercher les conditions de l'implication active des élèves dans des tâches signifiantes de pratique de la langue. Et dans ce domaine, la perspective actionnelle présente un intérêt indéniable et permet de créer une motivation réelle. Mais dans le contexte scolaire, ces tâches doivent être accompagnées si on veut qu'elles aient une efficacité en termes d'apprentissage, c'est-à-dire au niveau cognitif et psycholinguistique. C'est bien ce qu'écrivait déjà élisabeth Bautier en 1995 :

un enseignement de la langue qui se prend pour sa propre fin, c'est-à- dire qui institue la langue en objet et non le langage comme activité, peut être loin d'aider vraiment les élèves. Ce qui permet à un élève d'apprendre, de construire des savoirs, de rendre les activités scolaires signifiantes en tant qu'activités d'apprentissage, ce n'est pas la maitrise d'un système linguistique particulier mais celle de ses usages dans l'ensemble de ses fonctions, et en particulier de ses fonctions cognitives, et ce, quel que soit le système dans lequel elles se réalisent.

Il s'agit là, pour moi, d'un objectif fondamental qui, loin de rejeter les objectifs actionnels beaucoup plus locaux, devrait pouvoir les structurer, leur conférer une cohérence et une dynamique psycholinguistiques, mais à deux conditions: 1), que l'on admette que le temps de maturation important que nécessite cette dynamique est incompatible avec l'exigence d'immédiateté, et 2) qu'on prenne la peine de concevoir des programmes officiels où la composante grammaticale est structurée en fonction du rôle que les marqueurs peuvent jouer par rapport à chaque entrée actionnelle générique, et non, comme c'est le cas aujourd'hui, dans les nouveaux programmes pour les paliers 1 et 2, de façon globalement aléatoire, en fonction des exemples spécifiques de formulation qui sont donnés (pour une analyse plus approfondie des limites des programmes actuels, cf. Chini 2009a).

\section{Peut-on concilier les orientations sociolinguistique et psycholinguistique?}

17 Pour qu'un tel objectif soit possible, il conviendrait de sortir d'une vision trop souvent frontale des choses, où le continuum qu'implique la référence aux deux pôles, formel et anthropologique, du langage et donc de la linguistique se réduit à une simple opposition binaire entre ces deux pôles, conçus comme des positions de principe stables et antinomiques : soit on fait de la grammaire, soit on agit ; soit on réfléchit au système, soit on l'utilise ; ou encore soit on s'appuie sur la linguistique théorique et on ne peut que figer la langue dans les cadres formels qu'elle impose, soit on donne priorité au point de vue sociolinguistique et on laisse s'exprimer librement la variation langagière qui définit les pratiques. Sans doute cette présentation est-elle, elle aussi, caricaturale, mais elle me semble malgré tout faire écho à un type de représentation assez répandu, et en tout cas, sous-tendre la position rappelée en introduction, qui suggère une définition fort étroite de la linguistique théorique. S'il est vrai que jusqu'ici la dimension sociolinguistique était injustement laissée pour compte, il ne faudrait pas que sa valorisation entraîne un renversement du balancier au détriment de la dimension cognitive et psycholinguistique fondamentale de l'apprentissage. 

et, comme l'écrivaient déjà en 1997 Anne-Claude Berthoud et Françoise Demaizière, il faut traiter "les formes linguistiques tout à la fois comme traces des opérations de discours et comme indices de l'interprétation". Elles sont donc au cœur du travail langagier que doit effectuer le sujet. C'est pourquoi pour sortir de cette opposition binaire, il est essentiel de ne jamais occulter, dans la dynamique langagière, le troisième participant, celui qui fait le lien entre langue et pratiques, à savoir le sujet. Mais pour cela il faudrait revenir à une définition plus large que celle qui prime aujourd'hui : sujet social certes, mais aussi, en tant qu'être social collaboratif, sujet énonciateur, qui construit et interprète du discours, et, surtout dans la classe, sujet apprenant, c'est-à-dire sujet cognitif et psycholinguistique qui doit s'approprier les outils opératifs de cette construction et de cette interprétation. Cette centration sur le sujet, pris dans toute sa complexité, est, à mon sens, le seul moyen de tenir, en milieu scolaire du moins, tous les bouts de l'écheveau didactique, y compris celui qui mène à la tâche.

Si l'on reprend la classification proposée par Henri Portine, cela impliquerait d'opter pour une focalisation thématique sur la cognition, mais conçue non pas comme un domaine stable, mais comme le centre, le noeud de tous les rapports qui se jouent dans la classe, le "rapport apprenant-apprenant", mais aussi le "rapport au monde" intimement lié au rapport au langage, au travers de la construction de la langue étrangère et de son rapport avec la ou les langue(s) de référence du sujet, toutes choses, monde et langues, qu'il convient que l'apprenant conceptualise, si l'on veut qu'il puisse agir de façon autonome et efficace, en prenant en compte les données pragmatiques et sociales. Si effectivement avec une telle focalisation, on travaille sur "ce qui se passe dans la tête de l'apprenant", c'est afin de l'aider à construire tous ces rapports, qui sont fondateurs de ses compétences tout à la fois langagières, actionnelles et sociales, et qui, tous, qu'on le veuille ou non, ont une base cognitive et très largement psycholinguistique.

$C^{\prime}$ est bien dans ce sens-là, dès Charlirelle ${ }^{6}$, qu'a toujours été conçue, par les tenants de la didactique conceptualisante, la transposition, ou plutôt l'implication, dans le champ de la DLE, des concepts énonciatifs, concepts opératifs dont on estime qu'ils ont la potentialité d'aider, petit à petit, les apprenants à structurer leur perception de la langue étrangère de façon opérationnelle, et à les conduire, à terme, à dépasser l'irréductibilité des spécificités morphosyntaxiques de surface pour accéder à une perception des enjeux langagiers. Et c'est bien là que la linguistique, au sens où je l'ai définie, a un rôle essentiel à jouer. Tout d'abord, certes, en fournissant à la didactique ce qui est essentiel, comme le rappelle Henri Portine, à savoir: "le questionnement du linguiste", "une vision de l'activité langagière et des objets qu'elle manipule, une méthodologie d'approche", en d'autres termes la " conscience de la formulation des questions, (l') évolution des questionnements et (un) regard épistémologique". Ce rôle de l'apprenant comme linguiste en herbe a été largement exploré dans le cadre des travaux du Geped, je n'y reviendrai pas (cf. Chini \& Goutéraux, 2008). Mais, même si je suis d'accord avec Henri Portine pour dire qu'"il n'y a pas de traduction immédiate du savoir des linguistes en savoir-faire pédagogique", ce qui relèverait d'un applicationnisme étroit dont on a expérimenté les dangers, je veux croire que le savoir des linguistes peut être "un savoir procéduralisable". Comme le prouve l'expérience menée depuis plusieurs années par Line Audin et son équipe de l'INRP sur le concept <arb> qui fonde le modèle culiolien (cf. Audin, 2005 et 2008), je reste convaincue qu'une linguistique du sujet, pour moi prioritairement la linguistique de l'énonciation, mais pour d'autres cela peut être la linguistique cognitive (cf. par exemple, Lapaire \& Masse, 2006), peut 
jouer un rôle structurant, à condition d'admettre que le modèle de départ n'appartient pas aux seuls linguistes et peut être simplifié, adapté aux besoins du processus d'apprentissage, sans pour autant y perdre sa validité.

21 Mais en passant au service de la dynamique d'apprentissage, la linguistique doit muter. Les concepts linguistiques n'auront d'intérêt dans la classe que si, loin d'être des objectifs de contenu, ils se transforment en instruments psycholinguistiques, permettant au sujetapprenant, énonciateur et acteur social en puissance, de structurer ses connaissances en construction, en les mettant en rapport avec le déjà-là de sa ou ses langue(s) de référence (cf. Chini, 2004). C'est aussi sur cette base-là que devrait s'envisager un vrai plurilinguisme efficace (cf. Chini, 2009a, 2009b). Cependant, plus qu'un objectif absolu à atteindre, ce qui serait totalement utopique et ferait retomber la DLE dans l'applicationnisme, il s'agit en fait d'un choix épistémologique d'orientation des pratiques pédagogiques de la classe, qui n'exclut absolument pas la pratique, même actionnelle, mais fonde l'efficacité dans l'exécution de la tâche, et donc la qualité des résultats, sur la solidité du socle psycholinguistique, c'est-à-dire de la construction des compétences linguistiques et langagières.

\section{Retour sur la question de la norme et de la variation : le rôle essentiel de la compétence épi- / méta-linguistique}

Ces remarques nous ramènent à la question de la norme et de la variation. Tant historiquement que synchroniquement, il est indéniable que la variation est intrinsèque à la vie des langues et que toute norme qui vise à figer cette dynamique est appelée à être transgressée. Cependant, le fait même qu'un maximum de locuteurs se comprennent avec finalement un minimum de malentendus, qu'ils se reconnaissent comme appartenant à la même communauté linguistique, qu'ils puissent en général évaluer ce qui n'est pas ou plus une occurrence de leur langue, qu'ils puissent créer des formes signifiantes inédites qui ne soient pas aléatoires du point de vue morphosyntaxique ${ }^{7}$, et ce sans forcément avoir reçu une formation linguistique ou grammaticale particulière (on peut penser aux enfants, par exemple), l'ensemble de ces constats rend incontournable le fait que si variation il y a, elle n'est pas totalement libre et n'est pas seulement émergente et liée aux pratiques. Cette variation est cadrée, structurée, pour chaque individu, par la perception épilinguistique qu'il a des "possibles" de sa langue, et donc de ses limites, qui ne peuvent être fondés que sur un réseau opératif de relations formes-sens qui font système, en dehors de toute intervention de linguiste ou grammairien, relations qui sont tout à la fois différentes de celles qui structurent les autres langues, mais potentiellement compatibles avec elles (d'où le fait que l'on puisse traduire d'une langue à l'autre). En bref, qui dit variation, dit contrôle épilinguistique, essentiellement non conscient donc, sans lequel il devient impossible pour le sujet de percevoir la différence entre créativité, invention et dénaturation.

On connaît le rôle structurant de ces dimensions épi- et métalinguistiques dans la construction du langage chez l'enfant (cf. Gombert, 1990). Il est tout aussi essentiel dans l'apprentissage d'une langue étrangère. Ainsi, dans le cadre de ses travaux sur les effets de l'acquisition d'une langue additionnelle, Gessica de Angelis (2007:122) déclare ${ }^{8}$ : 
il est fort probable que la réflexion métalinguistique soit l'un des facteurs qui joue le rôle le plus important dans l'augmentation de la capacité des individus multilingues à apprendre de nouvelles langues. travers de l'interaction sociale en milieu naturel, particulièrement pour la langue première, nécessite une médiation explicite en contexte scolaire. On ne peut, en effet, dans ce contexte contraint, compter sur la richesse et le foisonnement des échanges pour faire percevoir les régularités sous la diversité discursive et susciter l'émergence d'une compétence épilinguistique qui parvienne à se démarquer de la référence de la langue première. Si les élèves doivent être sensibilisés à la variation et doivent être préparés à y entrer en tant qu'énonciateurs dans la langue étrangère, elle ne peut pour autant, dans ce contexte, être prônée comme première, si on ne veut pas rendre impossible toute appropriation cohérente du système étranger. Tout n'est pas possible, tout ne se vaut pas. D'un point de vue psycholinguistique, la variation découle de l'organisation interne de la langue, tout autant qu'elle la construit. Sans organisation, pas de variation signifiante. C'est pourquoi, en langue étrangère, pour compenser le déficit quantitatif et qualitatif de contacts et d'interactions, le contexte scolaire impose une stabilisation initiale liée à une organisation préalable progressive des données, qui, certes tendent à codifier la langue, mais restent une condition indispensable de sa construction par l'élève.

\section{Conclusion... ouverte}

En guise de conclusion, il convient de revenir aux questions soulevées au départ, en particulier celles de la place de la variation et du rôle de la linguistique. J'affirme donc que les langues étrangères sont bien "enseignables et apprenables", tout en respectant l'ouverture à la variation qui en fait des organismes vivants inscrits dans le temps. Mais j'affirme aussi, qu'en contexte scolaire extensif et contraint, c'est par le métalinguistique qu'on peut stimuler l'épilinguistique, et c'est par une certaine stabilisation initiale qu'on peut ouvrir sur la variation, une structuration et donc un recours stratégique à une certaine "norme" étant indispensables. Et dans cette opération stratégique, la linguistique théorique peut se révéler utile en proposant un cadre structurant efficace d'un point de vue psycholinguistique.

Mais, comme le montre l'échec de la greffe énonciative tentée par les programmes d'anglais pour le collège des années quatre-vingt-dix, cela ne va pas de soi (pour une analyse de cet échec, cf. Chini, 2004). Les obstacles sont nombreux et divers. Pour qu'une telle approche soit possible, il faudrait tout d'abord, comme indiqué plus haut, sortir de la logique binaire dans laquelle la DLE semble s'être enfermée (formes / pratiques, 
sociolinguistique / linguistique théorique, préoccupations sociolinguistiques / préoccupations psycholinguistiques, etc.), et concevoir des projets qui intègrent tous les aspects, sans exclusive. Il faudrait aussi clarifier la nécessaire adaptation aux contraintes du cadre scolaire des principes qui fondent la doxa actionnelle, si l'on ne veut pas que cela se fasse de manière un peu aléatoire et sauvage, comme c'est le cas aujourd'hui, et que l'on perde sur les deux tableaux de l'efficacité des pratiques et de la structuration psycholinguistique.

Mais il faudrait aussi faire évoluer les représentations réciproques que les enseignants et les linguistes, pas tous bien sûr mais beaucoup, ont du travail les uns des autres. Pour bien des linguistes, en effet, la didactique des langues est toujours vue soit comme un sousproduit, une version prétentieuse de la pédagogie, soit comme un domaine d'application, où la pureté de la théorie se dénature. Dans un cas comme dans l'autre, cela place tout échange de point de vue, tout essai de collaboration dans une impasse. Et pour ce qui est des collègues du secondaire, la longue pratique des préparations aux concours internes et externes m'a convaincue que le chemin de la sensibilisation à l'utilité de la linguistique est ardu, voire inaccessible pour beaucoup. Or pour qu'un enseignant puisse être un médiateur efficace dans ce domaine, il est indispensable qu'il ait lui-même réussi cette migration de l'épilinguistique au métalinguistique, dans les diverses langues qu'il maîtrise.

Considérons les jeunes collègues récemment issus des formations universitaires et dont on pourrait penser que la connaissance qu'ils ont acquis de la linguistique théorique au cours de leurs études devrait leur permettre non seulement de transférer à leur pratique d'enseignant les habitudes de réflexion linguistique et d'analyse des occurrences de discours qui étaient attendues d'eux en tant qu'étudiants, mais aussi de mener à bien une transposition efficace des concepts de la théorie linguistique afin d'en faire des outils structurants pour l'apprentissage. En fait il n'en est souvent rien et le problème est profond. Pour fonder une vraie formation à la linguistique et pas seulement en linguistique, vue comme un contenu, c'est-à-dire une formation qui tente de susciter des comportements nouveaux vis-à-vis du langage autant qu'elle cherche à faire acquérir des connaissances conceptuelles, il convient qu'un certain nombre de prérequis soient en place, en particulier les catégories syntaxiques et morphologiques de base qui permettent de percevoir rationnellement la linéarité de la langue comme un ensemble organisé, décomposable et analysable, ainsi évidemment que la perception du lien entre cette organisation formelle de surface et le sens qui se construit dans le discours. Or, pour la plupart des étudiants, cela n'est plus construit, même dans la langue de scolarisation, lors de leur cursus primaire et secondaire, en grande partie en conséquence de choix programmatiques qui, là aussi, ont, depuis des années, privilégié la pratique spontanée et l'oralité, autrement dit la productivité à court terme au détriment d'une structuration solide des connaissances linguistiques. C'est un constat que font, je pense, bien des enseignants du secondaire et du supérieur : les concepts grammaticaux classiques, dont il faut bien reconnaître le caractère indispensable et structurant comme point de départ de toute démarche théorisante de type métalinguistique, ne sont plus en place et il faudrait beaucoup plus de temps qu'on n'en a au cours des trois années de licence pour compenser ce déficit. Ce qui s'installe alors, au fil de semestres réduits à douze semaines ("grâce" à la réforme LMD, licence master doctorat), c'est une sorte de course contre le temps, totalement contraire aux exigences du processus de maturation. On passe très vite, trop vite, de la simple grammaire descriptive à la linguistique théorique, mais la terre n'ayant 
pas été assez préparée, fort peu de graines lèvent spontanément. Et pour les étudiants qui ne sont pas prêts, pour ceux qui ne sont pas en questionnement personnel, c'est-à-dire, malheureusement la majorité, la formation à la réflexion linguistique se résume à la mémorisation d'un discours magistral théorisant et au placage d'une métalangue, pour eux vidée de son sens, puisque le mot est venu avant la perception du concept et de son utilité explicative. La théorie comme somme de savoirs prime alors sur ce qui était visé, à savoir la linguistique comme procédure d'analyse, comme regard nouveau porté sur le langage. Pour les étudiants trop nombreux qui échouent, si la linguistique est bien ce " difficile trajet, chemin sans fin", dont parle Antoine Culioli (2009, quatrième de couverture) elle n'est que cela, et ne leur laisse jamais "entrevoir la belle et déroutante complexité du langage". Ces futurs enseignants ne passent jamais vraiment de l'épilinguistique au métalinguistique. Dans ces conditions, comment pourraient-ils, une fois enseignants, et sans véritable réflexion sur la transposition lors de leur formation professionnelle, faire fructifier l'apport de la linguistique, qu'il soit d'ordre conceptuel ou stratégique? Certains y parviennent certes, mais pour la majorité, une fois passé le concours, la linguistique, leur bête noire, est reléguée aux oubliettes avec toutes ces choses complexes et pompeuses qui n'intéressent que les universitaires. Ils se replient alors sur un service minimum grammatical qui finalement convient bien à la nouvelle doxa actionnelle. Doiton pour autant se résigner?

\section{BIBLIOGRAPHIE}

Angelis, G. de. (2007). Third or Additional Language Acquisition. Clevedon : SLA.

Audin, L. (éd.) (2005). Enseigner l'anglais de l'école au collège. Comment aborder les principaux obstacles à l'apprentissage. Paris : Hatier.

Audin, L. (2008). "Langues étrangères à l'école primaire : recherches INRP 1998-2003". In Chini, D. \& Goutéraux, P. Psycholinguistique et didactique des langues étrangères, numéro spécial des Cahiers de Recherche en grammaire anglaise de l'énonciation. Paris : Ophrys. pp. 143-154.

Bailly, D. (2008). "De Charlirelle au GEPED". In Chini, D. \& Goutéraux, P. Psycholinguistique et didactique des langues étrangères, numéro spécial des Cahiers de Recherche en grammaire anglaise de l'énonciation. Paris : Ophrys. pp. 253-255.

Bautier, É. (1995). Pratiques langagières, pratiques sociales. Paris : L'Harmattan.

Bernié, J.-P. (2003). "Langage pour apprendre, langage à apprendre et communautés discursives". In Chini, D. (coord.). Activité(s) en classe de langue - Actes du colloque de la journée d'étude du Geped, 24 janvier 2003, université Paris 7 Denis Diderot. pp. 16-32.

Berthoud, A.-C. \& Demaizière, F. (1997). "Des formes linguistiques pour communiquer : vers de nouveaux rapports entre linguistique et didactique." In Claude Springer (coord.). Les linguistiques appliquées et les sciences du langage - Actes du deuxième colloque de linguistique appliquée Cofdela. Strasbourg : Université Strasbourg 2. pp. 164-178. 
Bronckart, J.-P. (1996). Activité langagière, textes et discours : pour un interactionnisme socio-discursif. Lausanne : Delachaux et Niestlé.

Bronckart, J.-P. (2001). "S'entendre pour agir et agir pour s'entendre". In Baudouin, J.-M. \& Friedrich, J. (dir.). Théories de l'action et éducation. Bruxelles : De Boeck Université. pp. 133-154.

Castellotti, V. (dir.) (2009, à paraitre). Cahiers de l'Acedle, $\mathrm{n}^{\circ}$ 3. Charlirelle (1975-1980). Behind the words. Paris : OCDL Hatier.

Chini, D. (1997a). Quelques aspects de la problématique linguistique de l'enseignement de l'anglais dans le secondaire, évolution historique des textes officiels et courants actuels. Lille : Presses du Septentrion, thèses à la carte.

Chini, D. (1997b). "Application, transposition... ? Quels rôles pour la linguistique dans l'enseignement de l'anglais ? Les choix officiels et les choix de la recherche". In Claude Springer (coord.). Les linguistiques appliquées et les sciences du langage - Actes du deuxième colloque de linguistique appliquée Cofdela. Strasbourg : université Strasbourg 2. pp. 193-201.

Chini, D. (2004). Entre didactique et psycholinguistique, l'activité en classe de langue : quel rôle pour la problématique énonciative ? Note de synthèse pour l'HDR, université de Pau et des Pays de l'Adour. Disponible en ligne : http://acedle.org/spip.php?article210

Chini, D. (2008a). "Approche actionnelle, plurilinguisme et conceptualisation linguistique". In Chini, D. \& Goutéraux, P. (dir.). pp. 5-18.

Chini, D. (2008b). "Objectif méthodologique : construction d'une mémoire procédurale ?" In Chini, D. \& Goutéraux, P. (dir.). pp. 55-67.

Chini, D. (2009a, à paraître). "Premières rencontres avec la langue de l'autre : l'école primaire peut-elle construire les fondements d'une compétences plurilingue ?" In Gaspari, F. \& MarieLaverrou, F. Premières rencontres avec l'autre, revue Rives, cahiers de l'Arc Atlantique. Paris : L'Harmattan.

Chini, D. (2009b, à paraître). "Statut dominant de l'anglais : à propos de quelques effets sur les représentations et l'implication affective des élèves dans les apprentissages langagiers". Cahiers de l'Acedle, $\mathrm{n}^{\circ} 3$.

Chini, D. \& Goutéraux, P. (dir.) (2008). Psycholinguistique et didactique des langues étrangères, travaux du GEPED en hommage à Danielle Bailly, numéro spécial des Cahiers de Recherche en grammaire anglaise de l'énonciation. Paris : Ophrys.

Conseil de l'Europe (2001). Un cadre européen commun de référence pour les langues : apprendre, enseigner, évaluer. Paris : Didier.

Culioli, A. (2009). Variations sur la linguistique. Paris : Klincksieck. Nouvelle édition.

Forlot, G. (2009, à paraitre). "Place de l'anglais et paradoxes des apprentissages langagiers à

l'école". In Cahiers de l'Acedle, $\mathrm{n}^{\circ} 3$.

Gombert, J.-É. (1990). Le développement métalinguistique. Paris : PUF.

Lapaire, J.-R. \& Masse, J. (2006). La grammaire anglaise en mouvement. Paris : Hachette.

Morin, E. (2005). Introduction à la pensée complexe. Paris : Le Seuil. Nouvelle édition.

Pietro, J.-F. de, Matthey, M. \& Py B. (1989). "Acquisition et contrat didactique : les séquences potentiellement acquisitionnelles de la conversation exolingue". In Weil, D. \& Fugier, H. (dir.). Actes du troisième colloque régional de linguistique. Strasbourg, université des sciences humaines et université Louis Pasteur. pp. 99-124. 
Pietro J.-F. de \& Schneuwly, B. (1997). "Maîtriser un genre textuel en contexte scolaire, ou l'enseignement / apprentissage est-il une 'macro-séquence potentiellement acquisitionnelle' ?", Communication pour Rela (réseau européen de laboratoires sur l'acquisition des langues). Bâle, 15-17 septembre 1997.

Porquier, R. \& Py, B. (2004). Apprentissage d'une langue étrangère : contexte et discours. Paris : Crédif.

Portine, H. (2009). "Activités langagières, énonciation et cognition, la centration sur les apprentissages". Cahiers de l'Acedle, $\mathrm{n}^{\circ}$ 2. pp. 13-37.

Robillard, D. de (2009a). "Plurilinguismes et atomes crochus". Enregistrement vidéo de la journée Notions en Questions. Disponible en ligne : http://lettres.univ-tours.fr/82511382/0/ fichepagelibre/\&RH= - Consulté le 10/06/09.

Robillard, D. de (2009b, à paraître). "Plurilinguismes et atomes crochus". In Cahiers de l'Acedle, $\mathrm{n}^{\circ}$ 3 .

\section{NOTES}

1. Cf. l'introduction du texte de Gilles Forlot lors de la journée Notions en questions (NeQ) sur les plurilinguismes, à Tours en janvier 2009 (textes à paraître dans les Cahiers de l'Acedle, 2009, n 3, numéro dirigé par V. Castellotti) : "Si je commence par une brève mise au point épistémologique, je dirais que mon entrée dans la didactique est sociolinguistique. À vrai dire, elle ne peut qu'être de cette nature selon moi, dans la mesure où l'acte éducatif et l'acte pédagogique, dans le domaine des langues comme ailleurs, sont non seulement ancrés dans l'espace social mais contribuent à structurer celui-ci. Aussi, il n'y a pas pour moi de véritable didactique qui ne soit (en tout cas en partie) sociolinguistique".

2. On ne peut ainsi nier l'évolution historique de la linguistique depuis l'époque du structuralisme dominant et passer sous silence le rôle fondamental de la prise en compte des procédures et des opérations par les linguistiques du discours (ou du sujet) aujourd'hui dominantes dans le champ de la linguistique théorique, comme les théories de l'énonciation en France ou les linguistiques cognitives américaines, issues de la grammaire générative.

3. Cf. à titre d'exemples les colloques suivants: "Langue commune et changements de norme", organisé par l'équipe RES-Syled à l'université Paris III Sorbonne Nouvelle, en février 2009; "Inventive Linguistics", organisé par l'EA 741 de l'université Paul Valéry Montpellier III, en mars 2009 ; "Hétérogénéité et variation : quels objets socio-linguistiques et didactiques aujourd'hui ?", organisé par l'équipe Dipralang-Lacis à l'université Montpellier III, en mai 2009 ; "Canon scolaire et pratiques d'enseignement", journée d'étude organisée à l'IUFM de Lyon le 9 juin 2009 ; "Good usage and sociolinguistic variation: diachronic perspectives and national traditions", du 16 au 18 juillet 2009 à l'université de Cambridge, Murray Edwards College ; ou encore le numéro 6 de la revue Synergie à paraître en 2010 qui sera consacré au thème suivant: "Verbe, grammaire et enseignement : la prescription et l'usage".

4. Il précise : "Une didactique des langues a aussi sa place en linguistique dès lors que l'on considère que 'langues' est crucial dans le syntagme "didactique des langues"; ce qui ne signifie pas pour autant que la didactique des langues doive se restreindre à une pure application, mais qu'elle peut faire appel à des disciplines complémentaires comme l'ethnologie, les sciences de l'éducation, etc." Pour ma part, je serai plus réservée. Si bien entendu, l'objet de la didactique et celui de la linguistique sont sinon identiques du moins convergents, seules certaines problématiques de la DLE relèvent des sciences du langage et cette classification rend plus difficile la prise en compte, d'une part, des autres nombreuses convergences possibles, entre autres avec les différents champs relevant de la "culture", mais aussi avec les didactiques des autres disciplines, et surtout avec les sciences cognitives. L'importance accordée aux théories de l'action dans la perspective actionnelle 
actuelle prouve d'ailleurs le caractère central que peuvent prendre en DLE des problématiques non linguistiques. Henri Portine ajoute d'ailleurs immédiatement qu'il s'agit d'un "problème de centrage", d'un "choix sociopolitique" et que "la didactique des langues pourrait aussi se centrer sur les sciences de l'éducation. Dans ce cas, 'langues' perdrait de son acuité et la linguistique deviendrait une discipline complémentaire. Il faut choisir". Mais pourquoi faudrait-il choisir? Le champ de la didactique est, par définition, un champ complexe qui devrait s'appréhender comme tel, sans chercher à le rattacher prioritairement à une famille plutôt qu'à une autre (ce qui pourrait conduire à marginaliser celui qui n'opterait pas pour la centration dominante), mais en s'appuyant sur le principe de reliance, tel que défini par Edgar Morin, 2005, (cf. aussi l'usage qu'en fait l'équipe Aldidac, composante du CICC-EA 2529, dirigée par Joëlle Aden, dans le texte de cadrage du colloque de novembre 2009, à l'université de Cergy-Pontoise. NB. Texte disponible à l'adresse suivante: http://www.u-cergy.fr/rech/pages/aden/index.htm). Ce principe permet d'échapper aux catégorisations enfermantes et aux hiérarchies parfois arbitraires, pour mettre en avant les relations et les centrations multiples et interconnectées. C'est pourquoi, à mon sens, la didactique des langues doit continuer à affirmer son autonomie, seule solution pour faire reconnaître sa diversité, même si elle entretient des rapports privilégiés avec d'autres domaines, comme celui des sciences du langage. Libre ensuite à chacun de définir le point de vue qu'il privilégie pour tenter d'appréhender cette complexité, la diversité des problématiques didactiques rendant par ailleurs impossible une saisie holistique par un regard unique.

5. Je remercie Ruben Ibañez pour ce terme qu'il a explicité lors d'une discussion à l'occasion d'un séminaire du Geped en janvier 2009.

6. Charlirelle était un groupe de recherche en didactique et linguistique dont est issu le Geped et qui, dans les années soixante-dix, à l'institut d'anglais Charles $\mathrm{V}$ de l'université Paris 7 , réfléchissait à la façon de transposer la théorie des opérations énonciatives d'Antoine Culioli en une "grammaire vivante mise en cuvre en situation communicationnelle" (cf. Bailly, 2008) et qui a publié une méthode d'apprentissage de l'anglais, Behind the Words (Charlirelle, 1975-1980) destinée aux classes de collège, et qui proposait une approche conceptualisatrice, révolutionnaire pour l'époque, de la construction de la langue, fondée sur les concepts énonciatifs.

7. L'exemple donné à Tours par Didier de Robillard pour illustrer le caractère premier et constitutif de la variation est d'ailleurs exemplaire. Il s'agit de l'expression "bracelet de pied" créée pour rendre compte d'une réalité pour laquelle le locuteur en question n'avait pas de terme spécifique à disposition. Si cette expression est compréhensible par tout francophone c'est bien parce qu'elle respecte le fonctionnement syntaxique des constructions internominales du français. La réception aurait, n'en doutons pas, été beaucoup plus problématique, voire impossible, si le locuteur, d'origine anglophone ou germanophone, avait parlé de "pied bracelet", en optant pour le type de construction synthétique, typique de sa langue de référence, ou même s'il s'était contenté d'une juxtaposition de mots, "bracelet pied", en respectant l'ordre dominant "déterminé- déterminant" du français, mais sans recours à un marqueur de relation.

8. Notre traduction. Le texte original est le suivant: "metalinguistic thinking is likely to be among the most important factors that contribute to increase multilinguals's ability to learn languages". 


\section{RÉSUMÉS}

Cet article analyse l'évolution des relations entre linguistique et didactique consécutive à l'adossement de la DLE aux préconisations du CECR. L'orientation sociolinguistique marquée s'accompagne d'un recul des préoccupations plus étroitement linguistiques et psycholinguistiques, la priorité étant donnée, dans les pratiques, à l'immédiateté de la tâche et à la variation. Cela ne va pas sans poser problème dans le cadre scolaire, où le caractère "théâtral" des échanges s'accompagne naturellement d'une secondarisation des discours, et rend inévitable un recul métalinguistique à des fins de structuration psycholinguistique. Dans ces conditions, pour être pleinement efficace en contexte scolaire la perspective actionnelle doit intégrer une solide composante réflexive qui laisse le temps nécessaire à la maturation des connaissances construites, et, si possible, fondée sur un modèle psycholinguistiquement cohérent du système linguistique pouvant jouer un rôle utile dans la structuration cognitive des compétences.

The present article addresses the change in the relationship between the cognate fields of linguistics and language learning methodology under the influence of the CEFR. With the focus it puts on sociolinguistic variation and short term efficiency in accomplishing the task, it seems to have taken most of the light away from the problem of language building and psycholinguistic structuration. That tendency proves particularly problematic at school, where the "dramatized" use of the foreign language is bound to turn it into an object of discourse some time in the process of learning. That's why, to be really efficient at school, the action-oriented approach should be adapted to retain a fair amount of metalinguistic activities, language awareness and reflexive thinking, if possible based on a psycholinguistically coherent conception of the linguistic system to be acquired that could prove a necessary tool in the structuring process.

\section{INDEX}

Mots-clés : psycholinguistique, norme / variation, linguistique du sujet, perspective actionnelle, cadre scolaire

Keywords : psycholinguistics, norm/variation, subject-centered linguistics, action-oriented perspective, school learning environment

\section{AUTEUR}

\section{DANIELLE CHINI}

Danielle Chini est professeure de linguistique et didactique de l'anglais à l'université de Pau et des Pays de l'Adour, où elle est membre du laboratoire Langues, littératures et civilisations de l'arc Atlantique (EA 1925) au sein duquel elle est responsable du Geped (Groupe d'études en psycholinguistique et didactique). Dans la lignée des préoccupations de ce groupe, ses travaux portent essentiellement sur la dimension psycholinguistique de l'apprentissage des langues étrangères en milieu scolaire et sur le rôle central des procédures de conceptualisation. En 2008, 
elle a publié chez Ophrys, en collaboration avec Pascale Goutéraux de l'université Paris Diderot Paris 7, un ouvrage en hommage à Danielle Bailly, Psycholinguistique et Didactique des langues étrangères.

Courriel : danielle.chini[at]univ-pau.fr

Adresse : Université de Pau et des Pays de l'Adour, UFR LLSH, Avenue du Doyen Poplawski, 64013 Pau Cedex, France. 